\title{
rentrez: An R package for the NCBI eUtils
} API

\author{
by David J. Winter
}

\begin{abstract}
The USA National Center for Biotechnology Information (NCBI) is one of the world's most important sources of biological information. NCBI databases like PubMed and GenBank contain millions of records describing bibliographic, genetic, genomic, and medical data. Here I present rentrez, a package which provides an R interface to $50 \mathrm{NCBI}$ databases. The package is well-documented, contains an extensive suite of unit tests and has an active user base. The programmatic interface to the NCBI provided by rentrez allows researchers to query databases and download or import particular records into $\mathrm{R}$ sessions for subsequent analysis. The complete nature of the package, its extensive test-suite and the fact the package implements the NCBI's usage policies all make rentrez a powerful aid to developers of new packages that perform more specific tasks.
\end{abstract}

\section{Introduction}

The USA National Center for Biotechnology Information (NCBI) is one of the world's largest and most important sources of biological data. At the time of writing, the NCBI PubMed database provided information on 27.5 million journal articles, including 4.6 million full text records. The NCBI Nucleotide Database (including GenBank) had data for 243.3 million different sequences and dbSNP described 997.3 million different genetic variants. Records from all of these databases can be cross-referenced with the 1.3 million species in the NCBI taxonomy, and PubMed entries can be searched using a controlled vocabulary containing 272 thousand unique terms.

The NCBI provides access to a total of 50 databases through a web interface, public FTP sites and an API called Entrez Programming Utilities (eUtils, Sayers and Wheeler (2004)). R packages from the Bioconductor project (e.g., genomes, Stubben (2015); RMassBank, Stravs et al. (2013) and MeSHSim, Zhou and Shui (2015)) or available from CRAN (e.g., ape, Paradis et al. (2004); RISmed, Kovalchik (2017) and pubmed.mineR, Rani et al. (2014)) take advantage of the Eutils API to perform specific tasks. Two packages, rentrez and reutils (Schöfl, 2016), provide functions that cover the entire API. Here I describe rentrez, a package which provides users with a simple and consistent interface to eUtils. This paper discusses the design of the package, illustrates its use in biological research and demonstrates how the provided functions can aid the development of other packages designed to meet more specific goals.

\section{The eUtils API and rentrez}

The eUtils API provides endpoints for searching each of the databases it covers, finding cross-references among records in those databases and fetching particular records (in complete or summary form). The design of rentrez mirrors that of eUtils, with each of these endpoints represented by a core function that has arguments named to match those used in the API documentation (Table 1). The most important arguments to each $\mathrm{R}$ function are documented, and the help pages associated with these functions contain a reference to the relevant section of the eUtils documentation.

Table 1: Core eUtils endpoints and their rentrez counterparts

\begin{tabular}{lll}
\hline NCBI endpoint & Purpose & Core function \\
\hline esearch & Locate records matching search criteria. & entrez_search \\
elink & Discover cross-linked records. & entrez_link \\
esummary & Fetch summary data on a set of records. & entrez_summary \\
efetch & Fetch complete records in a variety of formats. & entrez_fetch \\
\hline
\end{tabular}

Typically, a user will begin by using entrez_search to discover unique identifiers for database records matching particular criteria. This function requires a database (argument $\mathrm{db}$ ) and a search term (argument term). The term argument can take advantage of the eUtils search syntax, in which queries can be associated with particular 'search fields' (enclosed in square brackets), to limit the number of matching records. The fields available for a given database can be retrieved with the function entrez_db_searchable. The following call demonstrates the use of this search syntax. This query finds scientific papers that contain the phrase 'R Package' in their title while limiting the result to include only papers published in 2017. 
pubmed_search <- entrez_search $(\mathrm{db}=$ "pubmed", term=" (R package[TITL]) AND 2017[PDAT]", use_history=TRUE)

pubmed_search

\#> Entrez search result with 62 hits (object contains 20 IDs and a web_history object)

\#> Search term (as translated): R package[TITL] AND 2017[PDAT]

For the most part, the objects returned by rentrez functions are S3 objects that inherit from "list" objects. Each of these classes has its own print method, providing users with a succint summary of the object's contents. In this case, entrez_search returns a an "esearch", object and the print method describes the number of records matching the query. These objects also contain either a character vector containing the unique identifiers of records that match the query or a "web_history" object that serves as a reference to this set of identifiers stored on the NCBI's servers. These "web_history" objects are useful when dealing with large numbers of records, as they reduce the number of identifiers that need to be exchanged between a user and NCBI servers. Both identifiers and "web_history" objects can be passed to the other core functions to retrieve information about the records they represent. For example, a call to entrez_summary returns information about each paper identified in the search above.

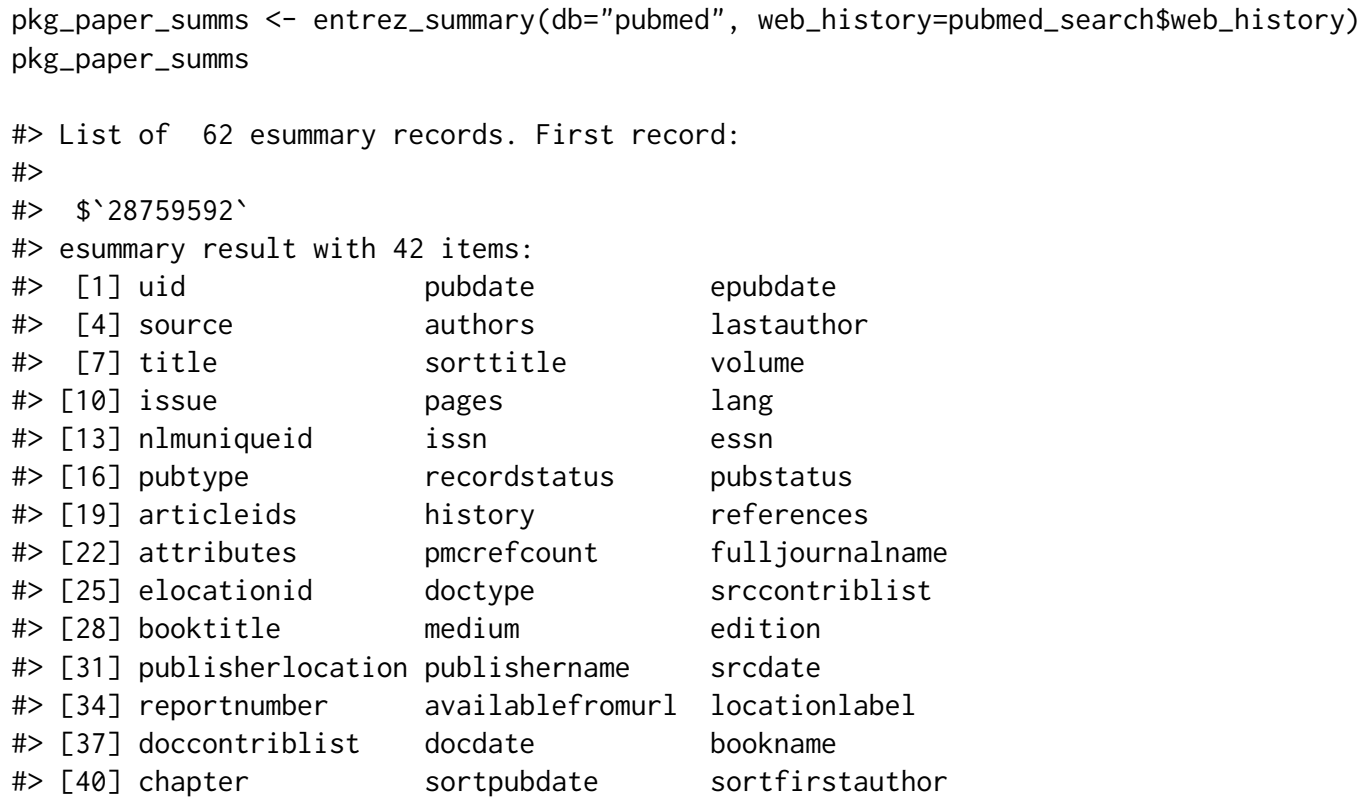

In addition to matching each of the eUtils endpoints, rentrez provides utility functions that facilitate common workflows. For example, the function extract_from_esummary allows users to extract some subset of the items contained in each of a set of summary records. In this case, the names of the journals that these papers appeared in can be retrieved. The resulting character vector can then be used to identify the PubMed-indexed journals that have published the most papers describing $\mathrm{R}$ packages this year.

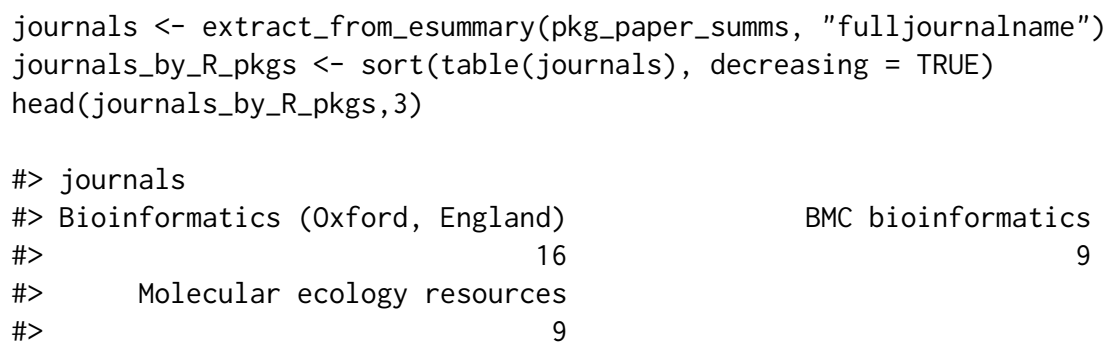

\section{Demonstration: retrieving unique transcripts for a given gene}

Records in the NCBI's various databases are heavily cross-referenced, allowing users to identify and download data related to particular papers, organisms or genes. By providing a programmatic interface to these records rentrez allows $R$ users to develop reproducible workflows that either 
download particular datasets for further analysis or load them into an R session. Here I demonstrate such a workflow, downloading DNA sequences corresponding to unique mRNA transcripts of a particular gene in a particular species.

Our aim is to retrieve the sequence of mRNA transcripts associated with the gene that encodes Amyloid Beta Precursor Protein in humans. This gene is identified by the gene symbol ${ }^{1}$ 'APP'. The NCBI database dealing with genetic loci (rather than particular sequences) is called 'Gene', so the first step to recovering the sequence data is discovering the unique identifier associated with APP in this database. This can be achieved with entrez_search, using the gene symbol and species in the search term.

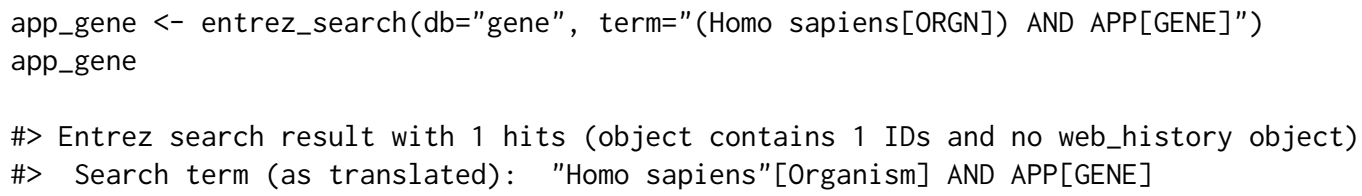

We now have a unique identifier for APP in the Gene database. In order to download sequences for this gene we need to find records from the NCBI Nucleotide database that are associated with the Gene record. The function entrez_link can be used to find cross-referenced records. In this case, a single call to entrez_link can identify human APP sequences in the nucleotide database in general and in a number of restrictive subsets of that database.

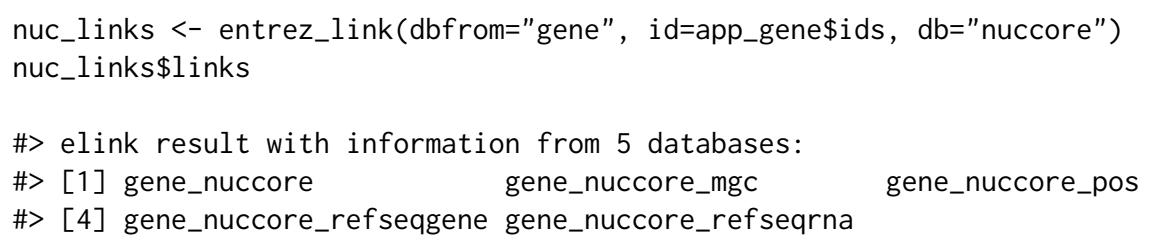

The RefSeq RNA subset on the Nucleotide database contains a curated set of mRNA transcripts for different genes. Thus the unique identifiers contained in the gene_nuccore_refseqrna element correspond to the sequences we wish to download.The function entrez_fetch allows users to retrieve complete records in a variety of formats. Here the sequences are retrieved in the standard 'fasta' format, and returned as a character vector with a single element.

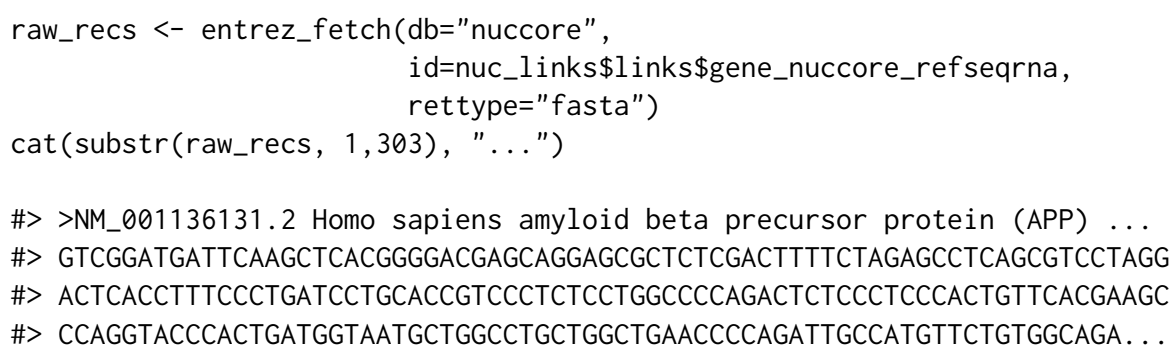

Sequences retrieved in this way could be written to file to be used by other software.

cat (raw_recs, file="APP_transcripts.fasta")

Alternatively, the sequences can be analysed within $\mathrm{R}$ using packages designed for sequence data. In this case, the data can be represented as a 'DNAbin' object using the phylogenetics package ape.

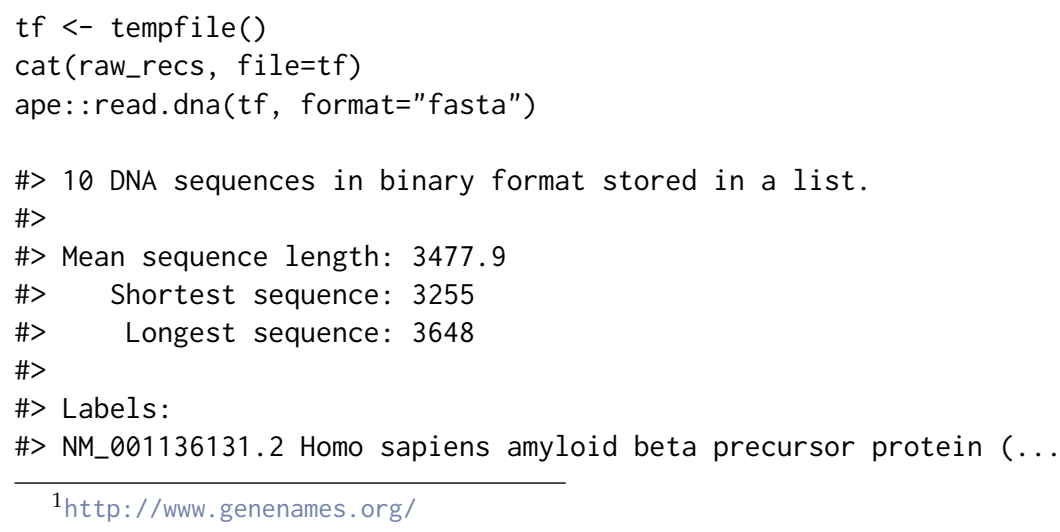


\#> NM_001136016.3 Homo sapiens amyloid beta precursor protein (... \#> NM_001204303.1 Homo sapiens amyloid beta precursor protein (... \#> NM_001204301.1 Homo sapiens amyloid beta precursor protein (... \#> NM_001204302.1 Homo sapiens amyloid beta precursor protein (... \#> NM_201414.2 Homo sapiens amyloid beta precursor protein (APP... $\#>$...

$\#>$

\#> Base composition:

\# $\quad$ a $\quad c \quad$ g $\quad t$

$\begin{array}{lllll}\#> & 0.276 & 0.223 & 0.258 & 0.244\end{array}$

The workflow detailed above provides a relatively simple example of how functions provided by rentrez can be used to identify, retrieve and analyse data from the NCBI's databases. The package includes an extensive vignette which documents each of the eUtils endpoints and demonstrates a number of detailed workflows. This document also describes how analyses can be scaled to analyse much larger datasets than are described in this paper. The tutorial can be accessed from within an $\mathrm{R}$ session by typing vignette(topic="rentrez_tutorial").

\section{Demonstration: development of a new package}

Development of rentrez has deliberately focused on producing a "low-level" package that provides a flexible interface to the entire eUtils API. As a result the package does not provide functions for any particular analysis or return records in any of the object classes made available for biological data by other packages. Rather, it is hoped that by providing a reliable interface to the eUtils API that meets the NCBI's terms of use rentrez will help other developers to build packages for more specific use-cases. Indeed, the package has already been used to integrate NCBI data into packages dealing with sequence analysis (genbankr, Becker and Lawrence (2017)), retrieval of phylogenetic trees (rotl, Michonneau et al. (2016)) and handling of full-text journal articles (fulltext, Chamberlain (2016)).

New packages that take advantage of rentrez will usually focus on providing a simple interface to users, so researchers will not need to be familiar with the syntax or arguments used in eUtils to perform tasks. Packages may also provide functions to parse files returned by entrez_fetch, either to extract particular information from those files or represent them as R objects. Here I present an example of a small package that performs both of these tasks. The software repository for this manuscript (https://github.com/dwinter/rentrez_ms) includes the code for a package called 'tidytaxonomy' that can be used to explore the taxonomic diversity of various NCBI databases. This demonstrates how the low-level code in rentrez can be used to develop specific applications that have a simpler interface than could be achieved with core rentrez functions alone.

The exposed functions from tidytaxonomy retrieve data from NCBI, but do not require the user to have any knowledge of the eUtils API. Internal functions

parse the XML formatted records returned from the NCBI Taxonomy database and extract relevant information. The core function tidy_taxonomy allows users to retrieve a part of the NCBI Taxonomy database in 'tidy data' format (Wickham, 2014).

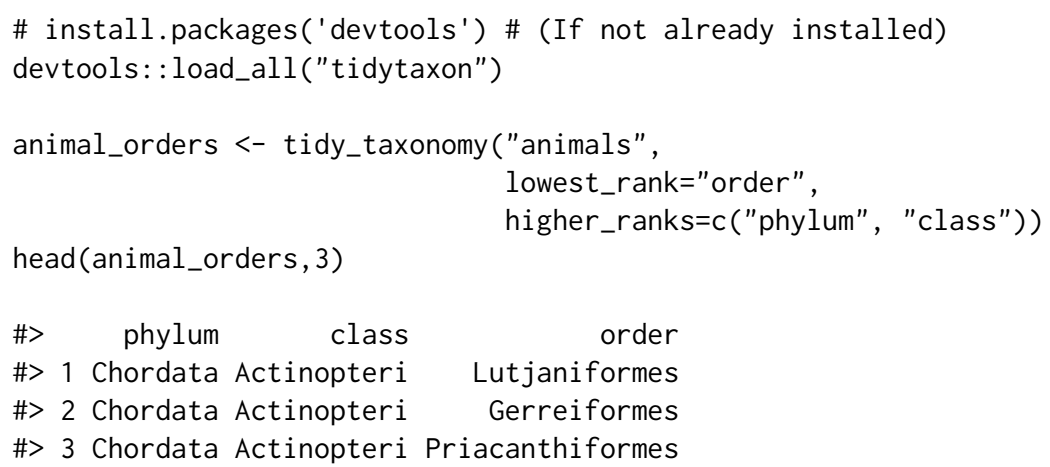

Once this data is obtained, two additional functions make it easy to include the number of records a given taxon has in a particular database. The function taxon_children is specifically for counting taxonomy records which are subordinate to a given taxonomic group. The other function, taxon_records, discovers records in any NCBI database.

animal_orders\$species <- taxon_children(animal_orders $\$$ order)

animal_orders\$genomes <- taxon_records (animal_orders\$order, db="genome") 

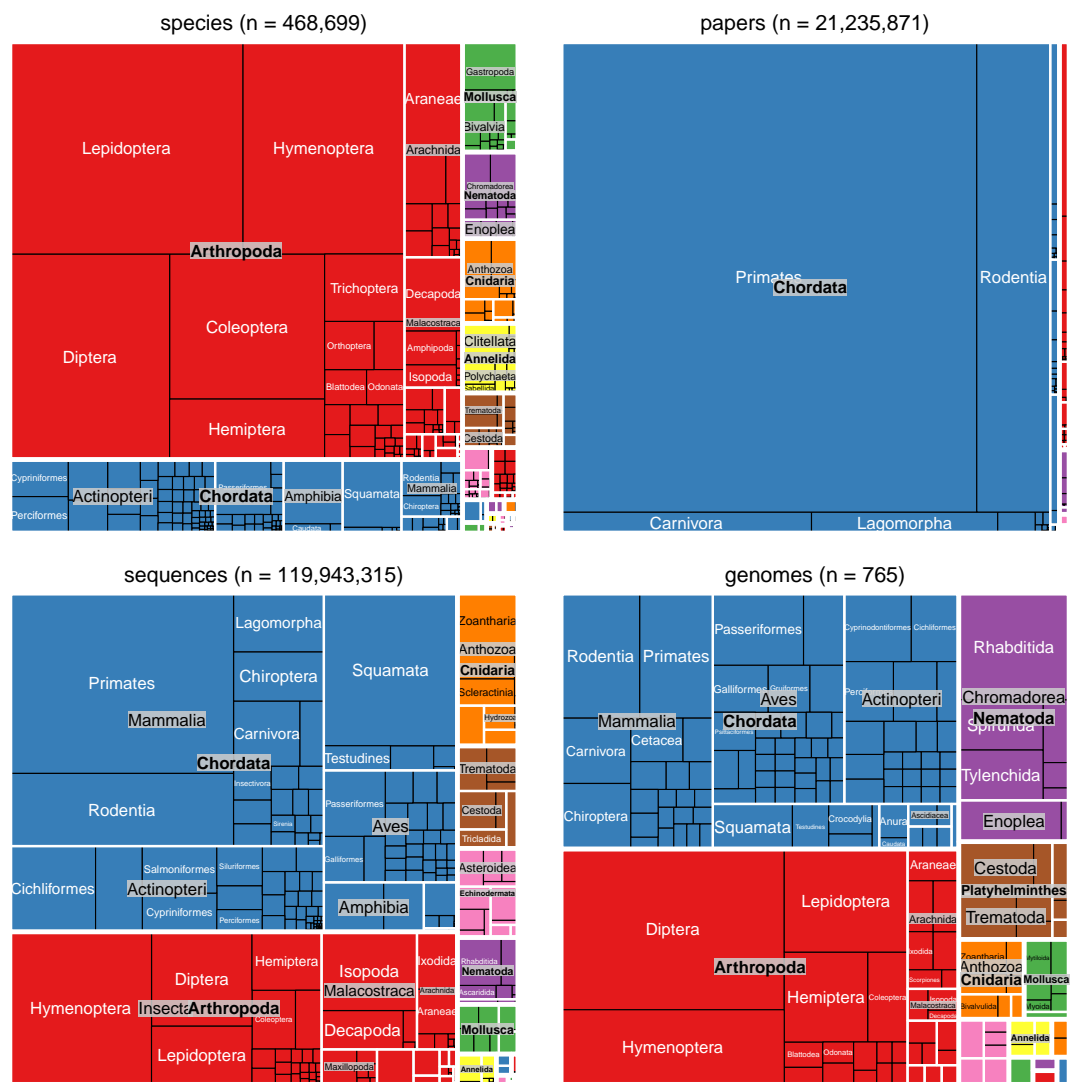

Figure 1: Taxonomic diversity of various NCBI databases (considering only animals). Panels in each plot are scaled to represent the number of database records corresponding to a given taxonomic rank and are shaded to reflect the phylum to which they belong. Starting from the upper left in clockwise direction subplots represent number of species in NCBI Taxonomy, the number of papers in PubMed, the number of sequences in NCBI Nucleotide database and the number of nuclear genome sequences in NCBI Genome database.

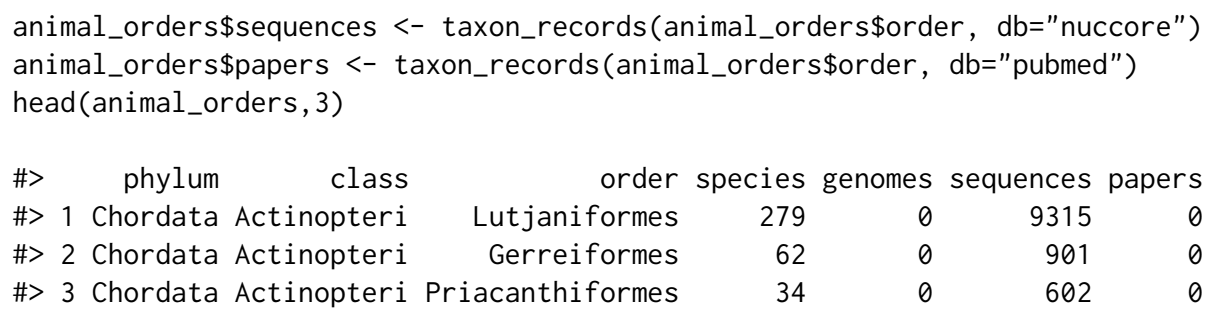

The resulting data can be used to visualise the taxonomic diversity of NCBI databases (Figure 1). The Appendix to this paper includes code that takes advantage of treemap (Tennekes, 2017) to produce these visualisations.

\section{Continued development of rentrez}

rentrez covers the complete eUtils API, is well-documented at the function and package level and includes an extensive test suite that covers internal functions as well as typical use-cases of the software. The current version of rentrez is thus considered a stable release, and it is unlikely any additional functionality will be added. The software is nevertheless still actively maintained to keep pace with CRAN and NCBI policies and to fix any bugs that arise. Software issues, including bug reports and requests for help with particular use-cases, are welcomed at the package's software repository: http://github.com/ropensci/rentrez. 


\section{Acknowledgements}

Development of rentrez has benefited greatly from being part of the rOpenSci project. I am especially grateful to Scott Chamberlain for his guidance. I am also very grateful to everyone who has provided pull-requests or filed issues including Chris Stubben, Karthik Ram, Han Guangchun, Matthew O'Meara, Reed Cartwright and Pavel Fedotov.

\section{Bibliography}

G. Becker and M. Lawrence. Genbankr: Parsing GenBank Files into Semantically Useful Objects, 2017. R package version 1.2.1. [p523]

S. Chamberlain. Fulltext: Full Text of 'Scholarly' Articles Across Many Data Sources, 2016. URL https: //CRAN.R-project.org/package=fulltext. R package version 0.1.8. [p523]

S. Kovalchik. RISmed: Download Content from NCBI Databases, 2017. URL https://CRAN. R-project. org/package=RISmed. R package version 2.1.7. [p520]

F. Michonneau, J. W. Brown, and D. J. Winter. rotl: An R package to interact with the open tree of life data. Methods in Ecology and Evolution, 7(12):1476-1481, 2016. URL https://doi . org/10. 1111/2041210x.12593. [p523]

E. Paradis, J. Claude, and K. Strimmer. APE: Analyses of phylogenetics and evolution in R language. Bioinformatics, 20:289-290, 2004. [p520]

J. Rani, S.Ramachandran, and A. R. Shah. An R Package for Text Mining of PubMed Abstracts., 2014. R package version 1.0.5. [p520]

E. Sayers and D. Wheeler. Building Customized Data Pipelines Using the Entrez Programming Utilities (eUtils). NCBI, 2004. [p520]

G. Schöfl. Reutils: Talk to the NCBI EUtils, 2016. URL https://CRAN. R-project. org/package=reutils. $\mathrm{R}$ package version 0.2.3. [p520]

M. A. Stravs, E. L. Schymanski, H. P. Singer, and J. Hollender. Automatic recalibration and processing of tandem mass spectra using formula annotation. Journal of Mass Spectrometry, 48(1):89-99, 2013. [p520]

C. Stubben. Genomes: Genome Sequencing Project Metadata, 2015. R package version 3.4.0. [p520]

M. Tennekes. Treemap: Treemap Visualization, 2017. URL https://CRAN.R-project.org/package= treemap. R package version 2.4-2. [p524]

H. Wickham. Tidy data. The Journal of Statistical Software, 59, 2014. URL http: //www. jstatsoft. org/ v59/i10/. [p523]

J. Zhou and Y. Shui. MeSHSim: MeSH(Medical Subject Headings) Semantic Similarity Measures, 2015. R package version 1.6.0. [p520]

David J. Winter

Institute of Fundamental Sciences, Massey University

Palmerston North 4442

New Zealand

ORCiD: 0000-0002-6165-0029

david.winteregmail.com

\section{Appendix}

Code used to produce Figure 1, using animal_orders data generated above.

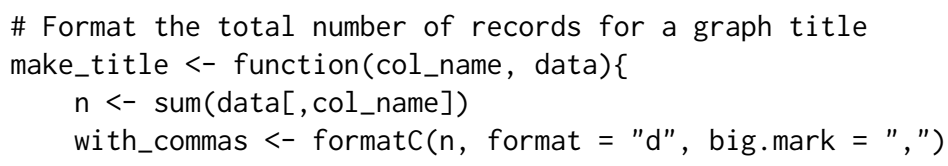


\}

paste0(col_name, " $(n="$, with_commas, ")")

\# Generate a treemap from taxonmic data.frame

\# * data= tidy_taxonomy data.frame

$\# *$ size_col $=$ name of column for $\mathrm{tm}$ tile-size

$\# *$ fill_col $=$ name of column for tile-fil

$\# \star$ row $=$ plot row in $2 \times 2$ grid

$\# *$ col $=$ plot col in $2 \times 2$ grid

$\# *$ pal $=$ palette for fill

taxic_diversity_tm <- function(data, size_col, fill_col, row, col, pal)\{ treemap (data,

index=c("phylum", "class", "order"), vSize=size_col, vColor=fill_col, palette=pal, type='categorical', position.legend="none",

title=make_title(size_col, data), border.col=c("white", "white", "black"), )

$\mathrm{vp}=$ viewport (layout.pos. row $=$ row, layout.pos.col $=\mathrm{col}$ )

\}

library (treemap)

library (grid)

library (gridExtra)

\# 24 phyla means some fill-colours will be re-used, ordering phylum factor by spp

\# will prevent any "major" phyla from getting the same colour.

spp_per_phylum <- aggregate(species phylum, FUN=sum, data=animal_orders)

phyla_ordered <- spp_per_phylum\$phylum[ order(spp_per_phylum\$species, decreasing=TRUE)]

animal_orders $\$$ phylum<- factor (animal_orders $\$$ phylum, levels=phyla_ordered)

pal <- $\operatorname{rep}($ RColorBrewer: :brewer.pal(8, name="Set1"), 3)

grid.newpage()

pushViewport $($ viewport $($ layout $=\operatorname{grid}$.layout $(2,2))$ )

taxic_diversity_tm(animal_orders, "species", "phylum", 1,1, pal)

taxic_diversity_tm(animal_orders, "papers", "phylum", 1,2, pal)

taxic_diversity_tm(animal_orders, "sequences", "phylum", 2,1, pal)

taxic_diversity_tm(animal_orders, "genomes", "phylum", 2,2, pal) 